\title{
In Loving Memory of Gulzar Ali
}

Waris M. Khuwaja

April 30th, 1995- just like any other day, Gulzar left his house with his friends to have some leisure time with them. Late that night, Gulzar's family got a call from a friend of Gulzar, stating that Gulzar had met with an accident and was in a well-known hospital in Karachi, an urban city in Pakistan. At the hospital, it was found that Gulzar had a compound fracture of his right leg and he was scheduled for an internal procedure to fix it. As any other hospital would do, the facility documented in the emergency room that he was allergic to penicillin. Soon after the surgery Gulzar became unconscious, and three days later his status was recommended as DNR, or Do-Not-Resuscitate, due to permanent brain damage. During his twenty-four hours of initial hospitalization, Gulzar, a young man in his thirties entered a vegetative state and spent the rest of his life in hospital. Soon after the incident, a root cause analysis was carried out. The root cause analysis indicated that the physician had prescribed $10000 \mathrm{u}$ of benzyl penicillin, the pharmacy had supplied streptomycin 10 Gm in place of benzyl penicillin, and the nurse had given those 10 vials stat IV (intravenous) in place of IM (intramuscular). This gross negligence by all three players, physician, pharmacist, and nurse, not only killed Gulzar but also victimized his whole family. At the time of this incident, Gulzar's youngest daughter was just nine months old. Due to the hospital error, all of Gulzar's responsibilities were placed on the shoulders of his wife, who was just thirty-four at that time. A young woman who had always been dependent on her husband had to become self-dependent. Along with the responsibilities of a father, Gulzar's wife also had to face tremendous hardships concerning her rights from her family.

On February 17th, 2008, Gulzar took his last breath. He choked while being fed his food through a feeding tube. During those thirteen years, Gulzar faced many hardships as a patient in hospital, but his family also faced some huge dilemmas. In 1995, when Gulzar began his new "vegetative" life in hospital, his family started a new life too, a life that was the opposite of what it used to be. Overnight, Gulzar's family lost possession of all the property that had been owned by Gulzar, because according to the rules in rural areas of Pakistan, the true inheritor of a father is his son. Gulzar had four daughters but no son. Gulzar's family was left with nothing but a few pennies. The family that had everything was reduced to nothing in just a day, but Gulzar's wife did not lose hope. As the hospital had admitted their mistake, Gulzar's family was compensated financially. However, that money was not enough to cover the living expenses for a mother and four daughters who had no other support. Also, this monetary compensation could never cover the

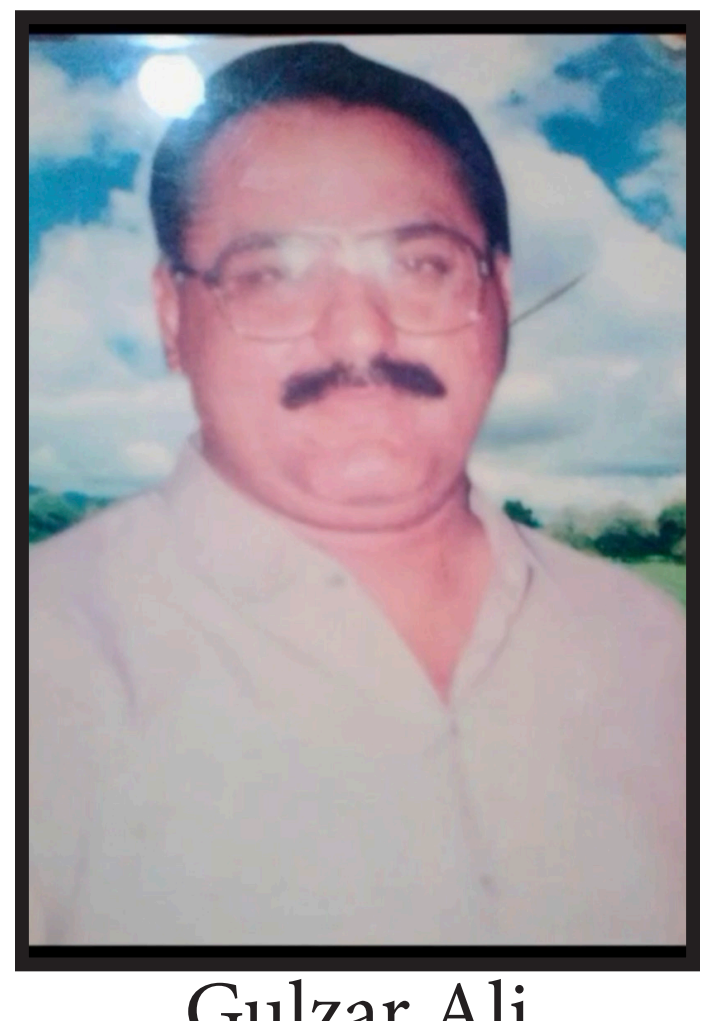

\section{Gulzar Ali}


cost of the dreams that Gulzar's wife had for her daughters, that they would become lawyers and doctors. Gulzar's wife soon began fighting for her right to the property of the husband she had lost. In 2005, Gulzar's wife started another battle in her life, and that was with breast cancer. After many years of struggles in hospitals and courtrooms, Gulzar's wife successfully won the battle with cancer and was able to get a small portion of Gulzar's property for her and her daughters. During his time in the hospital, Gulzar's three daughters got married and he became the grandfather of five children, including me.

Hospital error not only made Gulzar, my grandfather, vegetative for the rest of his life but also deprived a wife, a daughter, and a grandchild of the love of a spouse, parent, and grandparent. A hospital error turned over the lives of every member of Gulzar's family. This story serves as a great example of why every person that works in a healthcare organization bears a great responsibility for each and every patient. Every decision made in the healthcare organization about a patient's life not only affects that patient but also his or her whole family. Gulzar left us with a great example of this. Every healthcare professional should always keep in mind patient safety before making any decision and always abide by the Hippocratic Oath to treat each patient to the best of his or her ability, so that hospital errors are minimized as much as possible. 